\title{
Design of one-dimensional optical pulse-shaping filters by time-domain topology optimization
}

\author{
Yang, Lirong; Lavrinenko, Andrei; Hvam, Jørn Märcher; Sigmund, Ole
}

Published in:

Applied Physics Letters

Link to article, DOI:

10.1063/1.3278595

Publication date:

2009

Document Version

Publisher's PDF, also known as Version of record

Link back to DTU Orbit

Citation (APA):

Yang, L., Lavrinenko, A., Hvam, J. M., \& Sigmund, O. (2009). Design of one-dimensional optical pulse-shaping filters by time-domain topology optimization. Applied Physics Letters, 95(26), 261101.

https://doi.org/10.1063/1.3278595

\section{General rights}

Copyright and moral rights for the publications made accessible in the public portal are retained by the authors and/or other copyright owners and it is a condition of accessing publications that users recognise and abide by the legal requirements associated with these rights.

- Users may download and print one copy of any publication from the public portal for the purpose of private study or research.

- You may not further distribute the material or use it for any profit-making activity or commercial gain

- You may freely distribute the URL identifying the publication in the public portal 


\title{
Design of one-dimensional optical pulse-shaping filters by time-domain topology optimization
}

\author{
Lirong Yang, ${ }^{1, a)}$ Andrei V. Lavrinenko, ${ }^{1}$ Jørn M. Hvam, ${ }^{1}$ and Ole Sigmund ${ }^{2}$ \\ ${ }^{1}$ Department of Photonics Engineering, Technical University of Denmark, Ørsteds Plads 343, \\ DK 2800 Kongens Lyngby, Denmark \\ ${ }^{2}$ Department of Mechanical Engineering, Technical University of Denmark, Nils Koppels Allé 404, \\ DK 2800 Kongens Lyngby, Denmark
}

(Received 9 October 2009; accepted 7 December 2009; published online 28 December 2009)

\begin{abstract}
Time-domain topology optimization is used here to design optical pulse-shaping filters in $\mathrm{Si} / \mathrm{SiO}_{2}$ thin-film systems. A novel envelope objective function as well as explicit penalization are used to adapt the optimization method to this unique class of design problems. (C) 2009 American Institute of Physics. [doi:10.1063/1.3278595]
\end{abstract}

Shaping optical pulses into arbitrary waveforms is desirable in many applications including optical communication, nonlinear optics, and biomedical imaging. ${ }^{1}$ Different methods to design pulse-shaping filters are present today, based on various hardware configurations and design algorithms. Fourier synthesis methods are used by Weiner ${ }^{1}$ to design filters by distributing gratings and lenses. While having the benefits of incorporating programmable modules such as liquid-crystal spatial light modulators, this system is bulky, lossy, and difficult to integrate into waveguide systems. Fiber gratings have also become good candidates for shaping pulses. Many inverse design algorithms have been introduced, ${ }^{2-5}$ among which the inverse scattering layer peeling method (LPM) (Ref. 6) is the most used. However, LPM is only effective for designing one-dimensional (1D) fiber gratings for the following two reasons: (1) The resulting topology from LPM optimizations are with continuous index variations, which needs an extra synthesis method to be converted to feasible designs for two-phase platforms including thin-films and planar waveguides; ${ }^{7}$ (2) So far LPM has not been generalized to design two-dimensional (2D) structures. Hence new design methods have to be developed, if 2D filters are desired in planar waveguide systems.

Topology optimization (TO) is widely used in structural optimizations $^{8}$ and has in recent years been successfully adopted to optimize nanophotonic devices. 9 Various functions have been realized or improved in photonic-crystal based planar waveguides, including $90^{\circ}$ bends, ${ }^{10}$ Y-splitters, ${ }^{11}$ and slow-light couplers. ${ }^{12}$ The afore-mentioned optimizations are based on frequency-domain modeling and aim at improving the devices' bandwidths and/or transmissions. The benefits of time-domain optimizations have not yet been explored much. While the accuracy for frequencydomain analysis requires a broad range and fine intervals of the sampled frequencies, a single time-domain analysis can cover a wide frequency span with little error. This makes an intriguing difference in the consumptions of computational resources. Time-domain TO techniques have been utilized by Nomura ${ }^{13}$ in antenna design. A pilot study of a TO approach for single period pulses has also been carried out recently by Dahl. ${ }^{14}$ However, there has been no reports of using this new

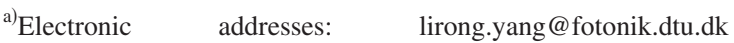

and optimization method to directly alter the temporal characteristics of a practical optical pulse which is an envelope containing rapid oscillations of the carrier wave. In our article, TO is combined with the finite-difference time-domain (FDTD) method to design 1D thin-film optical filters. Special treatments are made to make the inverse problem well-posed. The method is easy to extend to higher dimensional design problems, and makes good use of the versatility of FDTD in modeling various electromagnetic problems.

Consider a thin-film stack as the design domain for our optimizations. The whole domain is uniformly discretized into plates layered perpendicular to the propagation direction of the light, and is terminated using Mur's absorptive boundary conditions. The material of each plate is determined by design variable $\rho_{i}$ through linear interpolation

$$
\varepsilon_{r}^{i}=\varepsilon_{r_{1}}+\left(\varepsilon_{r_{2}}-\varepsilon_{r_{1}}\right) \rho_{i}, \quad\left(0 \leq \rho_{i} \leq 1\right) .
$$

In the above equation $\varepsilon_{r}^{i}$ is the relative dielectric constant of the $i$ th plate in the design domain, and $\varepsilon_{r_{1}}$ and $\varepsilon_{r_{2}}$ are the relative dielectric constants for the two design materials.

Different from minimizing the energy flux in the frequency-domain method, ${ }^{9}$ the objective function (OF), $F(\rho)$, for the time-domain pulse-shaping optimization should aim at minimizing the time-integrated squared difference between the output pulse $E_{\mathrm{o}}(t)$ and the prescribed pulse $g(t)$, namely,

$$
F(\rho)=\int_{t=0}^{T_{\max }}\left[E_{\mathrm{o}}(\rho, t)-g(t)\right]^{2} d t .
$$

There are two challenges in using the above formulation of OF. First of all, the optimization will be easily trapped in local minima due to rapid oscillations of the electric field. Consider a case where we want to delay a Gaussian pulse by simply increasing the dielectric constant of the bulk material [see Fig. 1(a)]. Figure 1(b) shows the objective function values as a function of $\tau$, which is the distance in time steps between the output pulse and the target pulse. For perfect overlap between the two pulses $(\tau=400)$, the OF value is zero. For $\tau$ different from 400, there are oscillations with local minima for each carrier wave period. The optimization is prone to be trapped at these minima, thus a new OF needs to be introduced to circumvent this problem. 
(a)

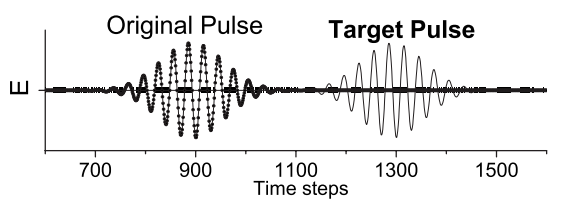

(b)

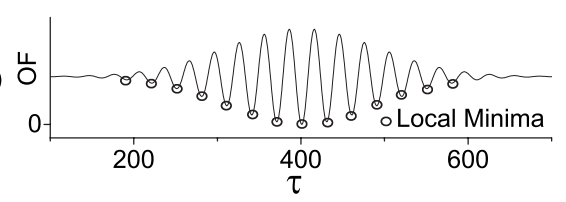

FIG. 1. A simple design case of delaying a pulse. (a) The electric field of the original (dotted) and target (smooth) pulses. (b) The OF values as a function of $\tau$, which is the number of time steps by which the original pulse is moved toward the target pulse.

Instead of optimizing the electric field directly as described in Eq. (2), we propose an envelope OF that optimizes the time-averaged electric field strength

$$
\hat{F}=\int_{t=0}^{T_{\max }}\left[\frac{\sum_{t^{\prime} \in N_{t}} E_{\mathrm{o}}^{2}\left(\rho, t^{\prime}\right)}{N}-\frac{\sum_{t^{\prime} \in N_{t}} g^{2}\left(t^{\prime}\right)}{N}\right] d t,
$$

where $N$ is the number of time steps in one carrier wave period. $N_{t}$ is the set of time points having the span of one carrier wave period and is centered at time $t$. By averaging the $E$ field within one period, the envelope is effectively extracted. The original inverse design problem is now reformulated into a more regularized one. Note, however, that since the envelope is being optimized now, the phase of the field will not be in direct control of the optimization anymore.

The second challenge is to ensure the fabricability of the optimized design. To comply with the gradient-based optimization algorithm, a continuous design variable $\rho$ is used. However, in order to fabricate the device on a two-phase material platform, a black and white design with reasonable minimum feature size has to be guaranteed. The linear interpolation relationship between the local material $\epsilon_{i}$ and design variable $\rho_{i}$ calls for extra measures to penalize intermediate values between 0 and 1 . The popular form of simplified penalization used in standard TO (Ref. 8) is not feasible here since it is difficult to impose a volume constraint on a scattering problem. Implicit morphological filters employed on densities ${ }^{15}$ are good candidates for minimum length scale control but not successful in penalizing the intermediate design variables entirely in our case. After various trials, it is shown that Borrvall and Petersson's ${ }^{16}$ explicit and meshindependent penalization imposed directly on the OF offers a good solution to our problem. The new OF will have the following form:

$$
\overline{\hat{F}}=\hat{F}+d \sum_{i} \widetilde{\rho}_{i}\left(1-\widetilde{\rho}_{i}\right),
$$

where the average density $\widetilde{\rho}_{i}$ is defined as $\widetilde{\rho}_{i}=\Sigma_{j \in D i} \rho_{j} / L . D_{i}$ is the vicinity of the $i$ th plate and $L$ is the length of $D_{i}$. The averaging of the densities works as a minimum length scale control to avoid generating too fine features in the optimized layout. Clearly the penalization term in Eq. (4) is minimum, only if all design variables are either 1 or $0 . d$ is a scaling factor that determines the extent to which intermediate values should be penalized. A too small $d$ would render the

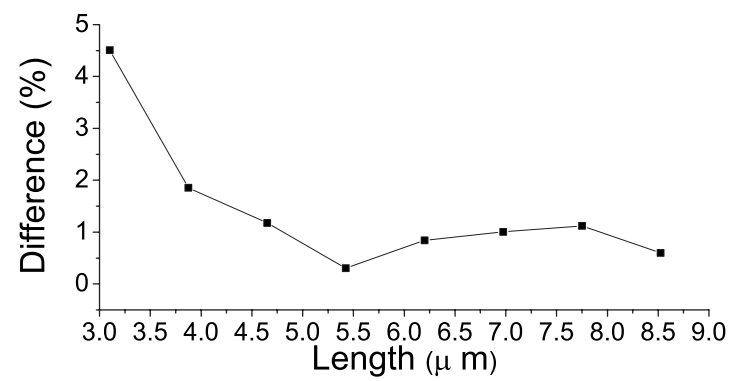

FIG. 2. The effectiveness of the optimization as a function of design domain size.

penalization ineffective, while a too large $d$ would cause the optimization to deviate from the original goal. Here, a continuation method was used where $d$ starts from $5 \times 10^{5}$ and gradually increases through the optimization iterations to shift the optimization focus from pulse shaping to black and white design.

Combining the above two alterations to the original OF, we now minimize the time-integrated envelope difference between the output and the target pulse plus the density penalization sum by varying the design variable $\rho$. In the meantime, Maxwell's equations should be obeyed as well as the upper and lower boundaries of the materials used for our system. The problem is thus formulated as

$$
\min _{\rho} \overline{\hat{F}}(\rho),
$$

subject to: Maxwell's equation,

$$
0<\rho_{i}<1, \forall i \text {. }
$$

The element-level sensitivities, which are the gradients of the OF with respect to each of the design variables, are key to the redistribution of the design materials. The adjointvariable analysis is developed by Niklova ${ }^{17}$ for the case of the FDTD method with the goal of minimizing the electric field. Only one extra system analysis is needed for deriving the exact sensitivities for all design variables. The first is the forward analysis yielding the response of the system while the extra adjoint analysis is carried out by swapping the input and output nodes and feeding the system with the adjoint current which depends on the field values from the forward analysis. For the envelope OF defined in Eq. (3), two system analyses are still sufficient to calculate the corresponding sensitivities. However, the adjoint current used in the second analysis has to be changed in order to adapt to the new OF. The penalization in Eq. (4) brings an additional sensitivity term which only has an explicit dependence on the design variable $\rho$. The sensitivities are then employed by the method of moving asymptotes ${ }^{18}$ to redistribute the material in the structure.

The optimization method is tested where an input Gaussian pulse with a full width at half maximum (FWHM) of 26 fs and a carrier wavelength of $1.55 \mu \mathrm{m}$ is to be converted to a square pulse in a stack of thin-film layers consisting of $\mathrm{Si}\left(\epsilon_{r}=12.3763\right)$ and $\mathrm{SiO}_{2}\left(\epsilon_{r}=2.3339\right)$. Due to the limited spectral range of the input pulse, the filtered square pulse will show overshoot and ringing known as the Gibbs effect. To comply with this mathematical limit, the target pulse is then generated by superimposing five Gaussian pulses with different time delays to mimic a square pulse so that its spec- 


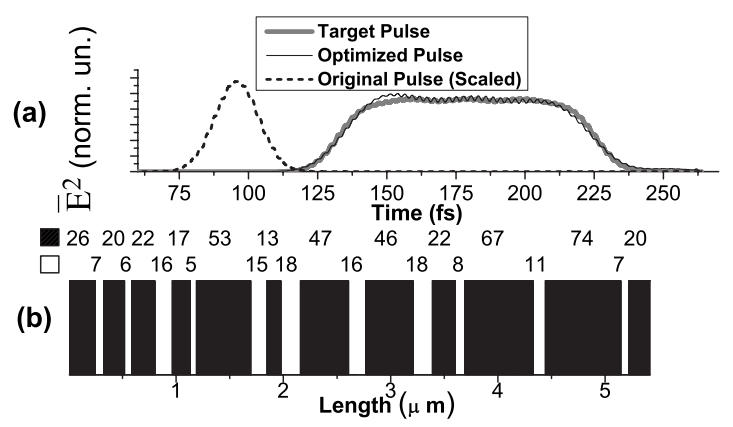

FIG. 3. Square pulse shaping. (a) The optimized output pulse with scaled amplitude (solid black) vs the target pulse (solid gray) as well as the scaled input Gaussian pulse (dotted black). (b) The optimized layout of the thinfilm pulse-shaping filter with $\mathrm{Si}$ (black) and $\mathrm{SiO}_{2}$ (white). The thickness of each layer is noted in terms of the corresponding number of grid points shown above the topology.

trum fits under that of the input pulse. The resulting target pulse has a FWHM of 90 fs with its amplitude decreased fivefold compared to that of the unfiltered pulse. The design domain is discretized into elements of the size of $\lambda / 165$, where $\lambda$ is the wavelength of the carrier wave of the pulses. Design domains with eight different lengths were optimized, each with 50 randomly distributed starting guesses to increase the chance of finding a solution close to global minimum. For the design domain of $5.42 \mu \mathrm{m}$ with 578 elements, a single optimization takes $12.6 \mathrm{~min}$ on an state-of-the-art single processor computer with the FORTRAN 95 code.

Figure 2 shows the optimization results in terms of the integrated difference between the optimized pulse envelope and the target pulse envelope. For each design domain size, a best result was picked among all 50 optimizations of different starting guesses. The optimized pulse approaches the target pulse better as the length of the design domain increases, since more reflectors are allowed in a longer device to tailor the pulse more accurately. This is a clear trend to be seen in the length between 3.10 and $5.42 \mu \mathrm{m}$, with the deviation from the target pulse down to $0.3 \%$ at the latter. However, if the design domain is too big, the optimization becomes more difficult due to the many more local minima introduced by the increasing number of design variables. The advantage of longer design domain is then lost, which can be seen after $5.42 \mu \mathrm{m}$.
The best design is that of $5.42 \mu \mathrm{m}$, or 3.5 times the wavelength of the carrier wave of the pulse. Figure 3(a) illustrates the optimized pulse (solid black) compared to the target pulse (solid gray) and the original Gaussian pulse (dotted black). The layout of such a pulse-shaping filter is depicted in Fig. 3(b). With the help of the explicit penalization term in Eq. (4), the thinnest layer in this structure is $65.8 \mathrm{~nm}$ which ensures fabricability.

It is shown in this letter that the proposed envelope OF combined with explicit penalization is a feasible way to adapt the FDTD-based TO to the optimization of the dynamics of optical pulses. This method can be directly extended to 2D/three-dimensional structures where planar waveguide systems can become potential candidates for more complex pulse shaping.

This work received support from the Eurohorcs/ESF European Young Investigator Award (EURYI) through the grant "Synthesis and topology optimization of optomechanical systems."

${ }^{1}$ A. M. Weiner, Rev. Sci. Instrum. 71, 1929 (2000).

${ }^{2}$ J. Skaar, B. Sahlgren, P. Y. Fonjallaz, H. Storoy, and R. Stubbe, Opt. Lett. 23, 933 (1998).

${ }^{3}$ J. Skaar and K. M. Risvik, J. Lightwave Technol. 16, 1928 (1998).

${ }^{4}$ J. Skaar, L. Wang, and T. Erdogan, IEEE J. Quantum Electron. 37, 165 (2001).

${ }^{5}$ N. Plougmann and M. Kristensen, Opt. Lett. 29, 23 (2004).

${ }^{6}$ R. Feced, M. N. Zervas, and M. A. Muriel, IEEE J. Quantum Electron. 35, 1105 (1999).

${ }^{7}$ J. Skaar, L. Wang, and T. Erdogan, Appl. Opt. 40, 2183 (2001).

${ }^{8}$ M. P. Bendsøe and O. Sigmund, Topology Optimization: Theory, Methods, and Applications (Springer, Berlin, 2003).

${ }^{9}$ J. S. Jensen and O. Sigmund, Appl. Phys. Lett. 84, 2002 (2004).

${ }^{10}$ J. S. Jensen, O. Sigmund, L. H. Frandsen, P. I. Borel, A. Harpøth, and M. Kristensen, IEEE Photon. Technol. Lett. 17, 1202 (2005).

${ }^{11}$ P. I. Borel, L. H. Frandsen, A. Harpøth, M. Kristensen, J. S. Jensen, and O. Sigmund, Electron. Lett. 41, 69 (2005).

${ }^{12}$ L. Yang, A. V. Lavrinenko, L. H. Frandsen, P. I. Borel, A. Tetu, and J. Fage-Pedersen, Electron. Lett. 43, 923 (2007).

${ }^{13}$ T. Nomura, K. Sato, K. Taguchi, T. Kashiwa, and S. Nishiwaki, Int. J. Numer. Methods Eng. 71, 1261 (2007).

${ }^{14}$ J. Dahl, J. S. Jensen, and O. Sigmund, Struct. Multidiscip. Optim. 36, 585 (2008).

${ }^{15}$ O. Sigmund, Struct. Multidiscip. Optim. 33, 401 (2007).

${ }^{16}$ T. Borrvall and J. Petersson, Comput. Methods Appl. Mech. Eng. 190, 4911 (2001).

${ }^{17}$ N. K. Niklova, H. Tam, and M. H. Bakr, IEEE Trans. Microwave Theory Tech. 52, 1207 (2004).

${ }^{18}$ K. Svanberg, Int. J. Numer. Methods Eng. 24, 359 (1987). 